\title{
Carcinoma de seno maxilar en paciente con liquen plano oral: Presentación de un caso clínico
}

\section{Maxillary sinus carcinoma in a patient with oral lichen planus: A case report}

\author{
Grando LJ*, Fabro SML*, Rath IBS*, Meurer MI*, Daniel FI**, Teixeira GV***, \\ Somacarrera $M L * * * *$
}

\begin{abstract}
RESUMEN
Mujer de 55 años de edad, fumadora, depresiva, con Liquen Plano Oral (LPO) de control clínico difícil, de más de 10 años de evolución. La paciente ha presentado períodos de remisión y exacerbación de las lesiones, de forma paralela presentó aumento de volumen intraoral en región maxilar izquierda, la superficie de la nueva lesión estaba ulcerada, y desprendía olor fétido en región alveolar sometida a exodoncias previas. El diagnóstico clínico inicial fue de una probable transformación maligna del LPO preexistente. Una biopsia incisional confirmó la presencia de carcinoma de células escamosas. Los exámenes tomográficos revelaron una amplia imagen destructiva dentro del seno maxilar, con zonas de erosión ósea en las estructuras adyacentes. Con la asociación de datos clínicos y de imagen llegamos al diagnóstico de carcinoma de células escamosas en seno maxilar. La paciente fue sometida a maxilectomía parcial y radioterapia adyuvante. Después de algunos meses, se manifestó la recidiva local con el agravamiento del cuadro clínico del paciente. Se instauró la quimioterapia paliativa adyuvante, sin éxito clínico. La paciente murió después de un año de tratamiento, debido a trombosis.

Palabras clave: Carcinoma de células escamosas, carcinoma de células escamosas en seno maxilar, liquen plano oral, diagnóstico oral, neoplasia de seno maxilar.
\end{abstract}

\section{SUMMARY}

Patient with difficult-to-manage oral lichen planus, with relapses and exacerbations over a 10 -year period, presented with a large ulcerative and foul-smelling intraoral mass in the left maxillary region. The initial clinical diagnosis indicated squamous cell carcinoma with possible association with previous history of lichen planus at the site. The incisional biopsy confirmed the presence of squamous cell carcinoma. The imaging exams revealed extensive involvement of the adjacent maxillary sinus cavity, with areas of bone erosion. The association of clinical, imaging and laboratory data indicated maxillary sinus carcinoma. The patient underwent maxillectomy and adjuvant radiation therapy. Local relapse and deterioration of symptoms occurred, and palliative chemotherapy was then used. The patient was refractory to chemotherapy and died one year later.

Key words: Squamous cell carcinoma, maxillary sinus carcinoma, lichen planus.

Fecha de recepción: 10 de octubre de 2012.

Aceptado para publicación: 12 de noviembre de 2012.

Grando LJ, Fabro SML, Rath IBS, Meurer MI, Daniel FI, Teixeira GV, Somacarrera ML. Carcinoma de seno maxilar en paciente con liquen plano oral: Presentación de un caso clínico. Av. Odontoestomatol 2013; 29 (4): 185-190.

Profesoras del Ambulatorio de Estomatología. Hospital Universitario, UFSC, Florianópolis, SC.

** Dentista, estagiário del Ambulatorio de Estomatología, del Hospital Universitario de UFSC, Florianópolis, SC.

*** Cirujano de Cabeza y Cuello. Centro de Pesquisas Oncológicas, CEPON. Florianópolis, SC.

**** Catedrática de Medicina Oral. Universidad Europea de Madrid. Madrid, ES. 


\section{INTRODUCCIÓN}

El Liquen Plano Oral (LPO) es una enfermedad mediada inmunológicamente y relativamente común que afecta a la mucosa oral y/o la piel (1). Los pacientes son generalmente adultos de edad media, teniendo una mayor predilección por las mujeres, en una proporción de 3:2 (1-4). El LPO puede presentarse de diferentes formas clínicas, y las formas reticular y ulcerativo/erosiva son los más frecuentes (3).

El tipo reticular es la forma más común de la enfermedad, usualmente asintomático y clínicamente se caracteriza por la presencia de Estrías de Wickham (4). El tipo ulcerativo/erosivo comúnmente causa dolor, llevando al paciente a la búsqueda de ayuda profesional; se manifiesta por presencia de zonas eritematosas, con distintos grados de ulceración y puede presentar las estrías de Wickham por la periferia de las úlceras. Usualmente las úlceras están localizadas en la mucosa yugal de forma bilateral, en los laterales y parte posterior de la lengua, en las encías, en el paladar y la mucosa labial (1). Algunos autores afirman que las lesiones son crónicas y la remisión espontánea es rara $(1,5)$.

No hay tratamiento definitivo para el LPO. El tratamiento paliativo tiene por objeto minimizar la sintomatología dolorosa, controlando las zonas de eritema y ulceración (4). Neville et al (2004) (1) afirmaron que no es necesario el tratamiento del LPO Reticular por no haber sintomatología y sólo se recomienda el seguimiento anual del paciente. Los tratamientos del LPO ulcerativo/erosivo pueden cambiar dependiendo de la intensidad de los signos y síntomas y de las condiciones generales de salud del paciente $(2,6,7)$. Consiste básicamente en la utilización tópica/local o por vía sistémica de diferentes tipos de corticosteroides $(8,9)$.

La Organización Mundial de la Salud define LPO como una condición cancerizable (10). Eisen y cols. (2000) (11) defendieron la influencia del estrés además del frecuente consumo de alcohol y tabaco en la exacerbación de las lesiones. Se considera bajo el riesgo de transformación maligna del LPO en carcinoma de células escamosas; sin embargo, la forma ulcerativa/erosiva muestra más propensión a tal $(3,4,12-15)$. Otros autores (9) destacaron la impor- tancia de la eliminación de factores que exacerban las lesiones, evitando el mordisqueo de la mucosa, reparando o cambiando restauraciones y prótesis en mal estado, manteniendo un programa de higiene oral, controlando el estrés y la dieta. La relación del LPO con lesiones malignas en otras regiones del cuerpo es poco probable.

La literatura indica que alrededor del tres por ciento de todos los cánceres de cabeza y cuello ocurren en el senos paranasales; esos, el $80 \%$, son del seno maxilar e, histológicamente, entre 60 y $90 \%$ representan el carcinoma de células escamosas (15). La mayoría de los cánceres de seno maxilar se diagnostican en fase avanzada debido a que el inicio de los síntomas no es específico y son similares a una sinusitis (16).

\section{PRESENTACIÓN DE UN CASO CLÍNICO}

Mujer de 55 años, fumadora, no ingesta de alcohol, con trastorno depresivo, acudió por primera vez al Ambulatorio de Estomatología del Hospital Universitario (Universidad Federal de Santa Catarina, Florianópolis, SC, Brasil) hace más de 10 años; la patología presentada fue diagnosticada como LPO y sus etapas sintomáticas fueron tratadas durante estos 10 años.

El LPO presentaba una variedad de aspectos clínicos, con áreas reticulares con estrías de Wickham asociadas con áreas eritematosas e inflamatorias en el paladar (Figura 1A). En las encías era más común la presentación en forma de placas blancas elevadas (Figuras 1B y 1C). En las figuras 1D y 1E se observan los tejidos obtenidos a partir de una biopsia incisional de la lesión, lo que confirma el diagnóstico clínico de LPO. Se ha postulado que las características principales del diagnostico histopatológico son hiperqueratosis y acantosis, degeneración hidrópica de la capa basal e infiltrado inflamatorio de linfocitos $\mathrm{T}$ en banda (Figuras 1D y 1E).

La paciente era seronegativa para la infección por el HIV y presentaba hepatitis B en su forma crónica, sin indicación de tratamiento. No había historia clínica familiar y las analíticas sanguíneas presentaban niveles adecuados de glucemia y colesterol. No había 
antecedentes médicos de aumento de la tensión arterial.

El equipo de estomatología solicitó una evaluación psiquiátrica y aconsejó a la paciente dejar de fumar; estos indicaciones nunca fueron seguidas por el paciente. Cuando aparecía sintomatología, se establecía el protocolo de tratamiento con enjuagues de corticosteroides tópicos de alta potencia (propionato de clobetasol a 0,05\%), habiendo remisión de las lesiones. Estos períodos de remisión eran coincidentes con la mejora de las condiciones psicológicas y con la disminución y/o con el cese temporal del tabaco. Las lesiones dermatológicas no estuvieron presentes en ningún período. La recurrencias eran frecuentes y por restricciones financieras, la paciente interrumpió el tratamiento muchas veces. La intensificación de los síntomas llevó a la prescripción de corticosteroides sistémicos (dexametasona $40 \mathrm{mg}$ / día). El uso sistémico desencadenó un síndrome de Cushing, con abrupta interrupción del tratamiento por iniciativa del paciente. Problemas familiares, incluyendo historia de violencia familiar, reducción del poder económico, un intento de suicidio por parte de su hija, entre otros, llevó al paciente a un marco psicoemocional extremadamente inestable, con el empeoramiento de las lesiones bucales.

El paciente dejó de acudir a sus revisiones de control clínico durante un año y volvió después de ser sometida a exodoncias de los dientes 25, 26 y 27 en otro servicio público de odontología. Hubo sintomatología dolorosa intensa y continuo olor fétido en la región operada. En el examen clínico, se observó la presencia de exudado purulento drenando por los alveolos. El diagnóstico clínico fue de osteomielitis posquirúrgica, tratada con antibióticos apropiados (amoxicilina $750 \mathrm{mg}$ tres veces al día), pero el cuadro clínico evolucionó rápidamente en pocas semanas con la aparición de una ulceración exofítica en la región alveolar operada (Figuras 2A y 2B), acompañado de dolor intenso y espontáneo. Bajo la sospecha clínica de transformación maligna del LPO, la ulceración fue biopsiada con urgencia. El diagnóstico histopatológico fue de carcinoma invasivo espinocelular, con moderado grado de diferenciación (Figura 3). Los exámenes radiológicos por tomografía computarizada mostraron una amplia masa tumoral ya invadiendo el seno maxilar y el suelo de la órbita izquierda (Figura 2B), lo que definió el diagnóstico de carcinoma espinocelular en seno maxilar.

El paciente fue derivado a un centro de referencia para tratamiento de pacientes con cánceres. El estadio clínico fue T4NOM0 y el tratamiento propuesto fue maxilectomía total con preservación de la órbita izquierda (Figura 2C) sin vaciamiento cervical. Debido al compromiso de los márgenes de la resección quirúrgica, se ha decidido hacer la radioterapia adicional (dosis total $=7.360 \mathrm{cGy}$ ). El tumor resultó ser resistente a las radiaciones y a las quimioterapias paliativas. El paciente tuvo recurrencia local en la cara y una supervivencia de un año (Figura 2D), falleciendo como consecuencia de una trombosis.

Durante el tratamiento, la paciente firmó el consentimiento informado, autorizando la publicación de su historia y de sus imágenes.

\section{DISCUSIÓN}

El caso clínico presentó la coincidencia de LPO y un carcinoma de seno maxilar en el mismo paciente. Inicialmente la manifestación de las dos patologías juntas generó un factor de confusión diagnóstica, apareciendo como dos lesiones independientes. El bajo seguimiento de las recomendaciones terapéuticas por parte del paciente, el hábito tabáquico continuado y sus numerosos problemas personales, sin duda, dificultaron el control del LPO.

La aparición de una ulceración poco tiempo después de las exodoncias retrasó el diagnóstico (Figura 2A). Fue imposible realizar análisis histopatológicos de la mucosa del seno maxilar para determinación de presencia del LPO, al encontrarse muy destruida la misma. Este hecho, posiblemente, podría aclarar las preguntas sobre la posible implicación de la mucosa nasal por el liquen plano oral (8).

Las imágenes de tomografía computarizada fueron esenciales para aclarar el diagnóstico (Figura 2B). El hecho de que el seno maxilar estuviera ya casi en su totalidad afectado por la lesión (Figura 2B) y los análisis histopatológicos (Figura 3) llevaron a el diag- 
nostico de Carcinoma Espinocelular de Seno Maxilar. El diagnóstico de este carcinoma usualmente es tardío por cuestiones anatómicas; el seno maxilar es una cavidad que ofrece poca resistencia al crecimiento del tumor, y los signos y síntomas son inespecíficos y ocurren tardíamente (16). La relación de proxi- midad del seno maxilar con zonas nobles (base de cráneo, órbita, cavidad nasal, seno esfenoidal, y seno etmoidal frontal) hace que la cirugía sea un procedimiento complejo y mutilante, lo que dificulta la rehabilitación (6). Según Paulino et al (1998) (6), son poco comunes las manifestaciones intraorales de los car-

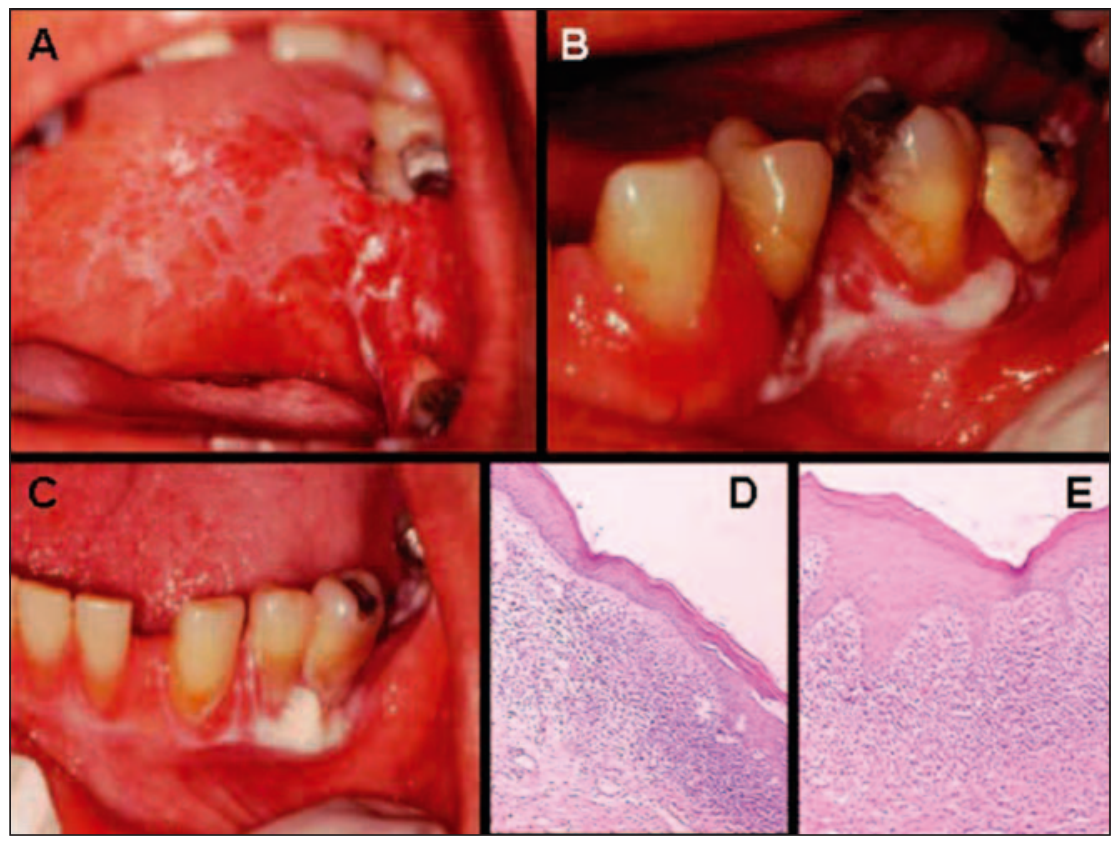

Fig. 1. A: LPO reticular con las estrías de Wicham intercaladas con regiones erosionadas en paladar duro; $\mathbf{B}$ y C: LPO en la forma de placas blancas en la mucosa de revestimiento alveolar; D y E: Las características histopatológicas con atrofia epitelial, hiperqueratinización, degeneración hidrópica de la capa basal epitelial y proceso inflamatorio crónico en banda, con un predominio de linfocitos en la lámina propia. Coloración HEE.
Fig. 2. A: Presencia del úlcera de carcinoma en la región alveolar superior izquierda; B: Tomografía computarizada muestra la presencia de gran masa tumoral en la región de seno maxilar izquierdo; C: La cara del paciente con postoperatorio de 10 días; D: Lesión exteriorizada del tumor en la cara del paciente en la fase terminal.

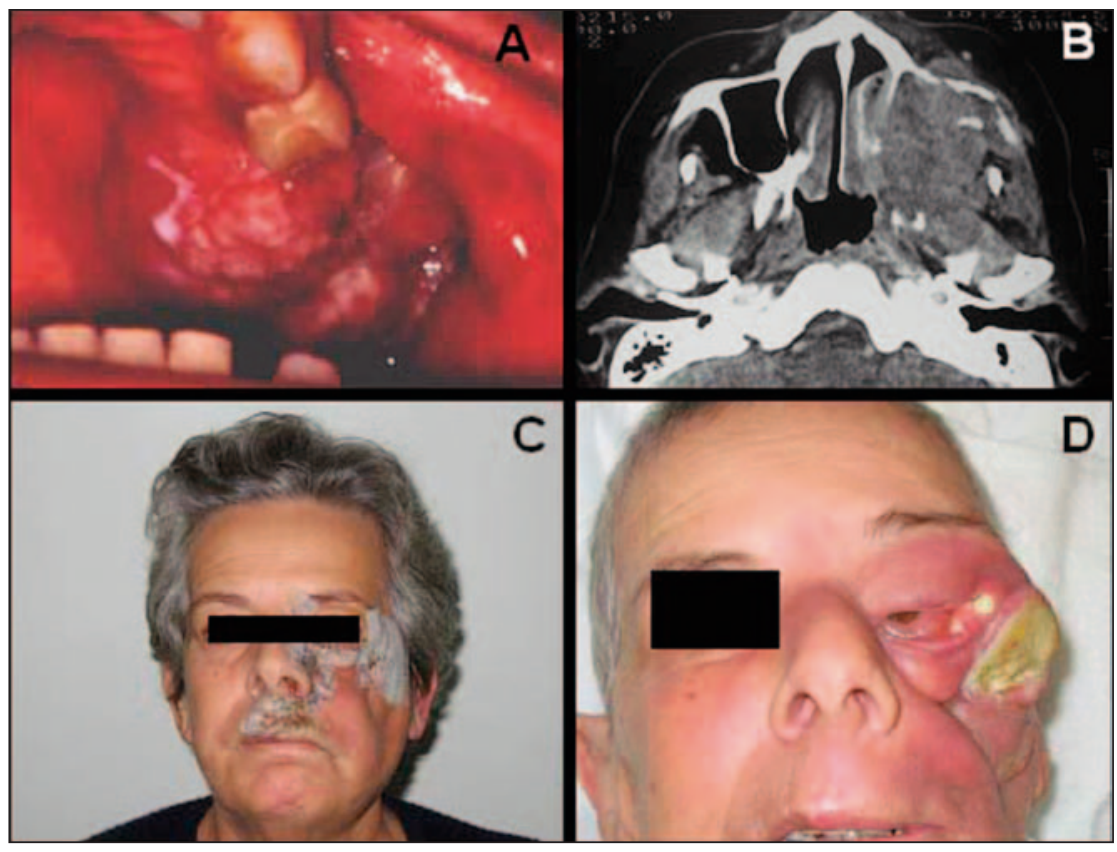




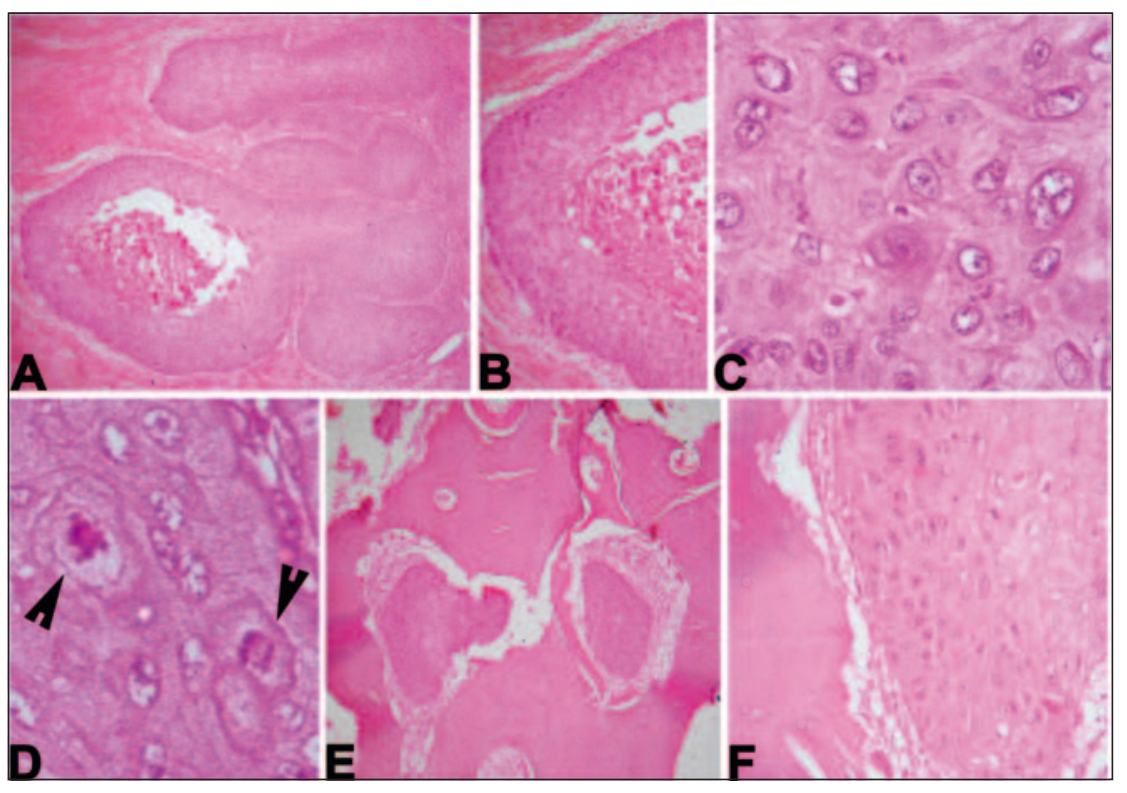

Fig. 3. A: Las de células epiteliales neoplásicas; B: Detalle de A; C: Pleomorfismo celular y nuclear; D: Las flechas muestran imágenes de mitosis atípica; E: La agresividad de la lesión al tejido óseo adyacente; F: El mayor aumento de E. Coloración H E E. cinomas de seno maxilar, siendo más común el compromiso de la órbita, cavidad nasal, seno etmoidal y área pterigoidea.

El tratamiento de elección para la mayoría de los casos de carcinoma de células escamosas del seno maxilar implica la cirugía y la radioterapia, con o sin quimioterapia asociada (5-7) La maxilectomía total con o sin extirpación de la órbita es una cuestión importante debido al compromiso estético y facial del paciente, puede causar importantes secuelas funcionales. Algunos cirujanos prefieren no remover la órbita y hacer uso de la radioterapia coadyuvante para complementar el tratamiento del resto del tumor (7); debe considerarse que el mayor fracaso en el tratamiento (16) del carcinoma de seno maxilar es la recurrencia en el mismo sitio primario de la lesión. Por lo tanto, el factor más importante en el tratamiento de estas lesiones que impliquen el seno maxilar es la prevención de la recurrencia local, que varía de 35 a $80 \%$ dependiendo de cada estudio $(15,16)$. Choi et al (2004) (15) informaron que el $80 \%$ de estos tumores tienen recurrencia local un plazo de dos años después de la cirugía. Las tasas de supervivencia de estos pacientes libres de enfermedad después de 3, 5 y 10 años son largas $(16,17)$.

Para el caso que hemos presentado se eligió el tratamiento quirúrgico, debido a la falta de metás- tasis regionales cervicales o a distancia, de modo similar a la que se discute en otros estudios $(6,15)$. La histopatología de los márgenes quirúrgicos quedó comprometida, siendo obligatorio el uso de la radioterapia complementaria, a pesar de que la literatura es controvertida sobre sus beneficios verdaderos $(2,6,7,15)$. La opción por el uso de la quimioterapia paliativa objetivó la estabilidad del cuadro clínico durante más de tiempo y la prevención de la enfermedad metastásica. Debido al gran deterioro funcional, el panorama empeoró y el paciente murió de trombosis después de aproximadamente un año.

\section{CONCLUSIÓN}

En el caso presentado, creemos que las lesiones LPO y Carcinoma Escamoso de Seno Maxilar se han producido de forma independiente. El retraso en el diagnóstico de carcinoma de seno maxilar sin duda contribuyó a la rápida evolución del paciente a la muerte.

\section{AGRADECIMIENTOS}

Agradecemos a la profesora doctora Elena Riet Correa Rivero toda su ayuda con las fotomicrografías; 
agradecemos a los académicos de Odontología, Felipe Perozzo Daltoé y Patrick Venturini, la atención odontológica con el paciente.

\section{BIBLIOGRAFÍA}

1. Neville WB. et al. Patol Oral \& Maxilofacial 2004; 2.ed. Rio de Janeiro: Guanabara Koogan.

2. Petti PF, Bagán JV, Scully C, Chaparro N. Transformación Maligna del Liquen Plano Oral en Tres Nuevos Casos. Acta Otorrinolaringol Esp 2004;55 (1):41-4.

3. Silverman S Jr. Oral Lichen Planus: A Potentially Premalignant Lesion. Journal of Oral and Maxillofacial Surgery 2000;58(11):1286-8.

4. Sugerman PB, Savage NW. Oral lichen planus: Causes, diagnosis and management. Austral Dent J 2002;47,4:290-7.

5. McCreary C E, McCartan B E. Clinical management of oral lichen planus. British J Oral \& Maxillofacial Surgery 1999;37(5):338-43.

6. Dulguerov P, Jacobsen MS, Allal AS, Lehmann W, Calcaterra T. Nasal and paranasal sinus carcinoma: are we making progress? A series of 220 patients and a systematic review. Cancer 2001; 92(12):3012-29.

7. Kawashima M. Influence of Postsurgical Residual Tumor Volume on Local Control in Radiotherapy for Maxillary Sinus Cancer. Japan J of Clin Oncology 2001;31(5):195-202.

8. Blanco Carrión A, Otero Rey E, Peñamaría Mallón M, Diniz Freitas M. Diagnóstico del líquen plano oral. Av Odontoestomatol 2008;24(1):11-5.

9. García-Pola Vallejo MJ, García Martín, JM. Tratamiento del líquen plano oral: una revisión. Av Odontoestomatol 2008;24(1):45-48.

10. Hietanen J. A retrospective study of oral lichen planus patients with concurrent or subsequent development of malignancy. Oral Oncology 1999;35(3):278-82.

11. Eisen D. The clinical features, malignant potential, and systemic associations of oral lichen planus: a study of 723 patients. J Am Acad of Dermatology 2002;46(2):207-14.

12. Cowan CG, Gregg TA, Napier SS, Mckenna SM, Kee F. Potentially malignant oral lesions in northern Ireland: a 20-year population-based perspective of malignant transformation. Oral Dis 2001;7(1):18-24.

13. Mignogna MD. et al. Clinical behaviour of malignant transforming oral lichen planus. Europ J Surg Oncology 2002;28(8):838-43.

14. Mignogna Michele D. et al. Immune activation and chronic inflammation as the cause of malignancy in oral lichen planus: is there any evidence? Oral Oncology 2004;40(2):120-30.

15. Van Der Meij EH. et al. The possible premalignant character of oral lichen planus and oral lichenoid lesions: A prospective study. Oral Surg, Oral Med, Oral Pathol, Oral Radiol, and Endodontology 2003;96:164-71.

16. Choi EC, Choi YS, Kim CH, Kim K, Kim KS, Lee JG, Kim GE, Yoon JH. Surgical outcome of radical maxillectomy in advanced maxillary sinus cancers. Y Med J 2004;45(4):621-8.

17. Paulino AC. et al. Results of Treatment of Patients with Maxillary Sinus Carcinoma. Cancer 1998; 83 (3):459-65.

18. Edwards PC, Kelsch R. Oral Lichen Planus: Clinical Presentation and Management. J of the Can Dent Assoc 2002; 68(8):494-9.

\section{CORRESPONDENCIA}

María Luisa Somacarrera Pérez

Avenida de América, 37

28002 Madrid

Correo electrónico: mluisa.somacarrera@em.es 